\title{
Editorial
}

\section{Minimally invasive spinal deformity surgery}

Christopher I. SHAFFrey, M.D., and Justin S. Smith, M.D., Ph.D.

\footnotetext{
Department of Neurological Surgery, University of Virginia Health System, Charlottesville, Virginia
}

Numerous publications have demonstrated a close relationship between sagittal spinopelvic alignment and health-related quality of life (HRQOL) outcomes after adult deformity surgery. ${ }^{3,4,7}$ More recently, the relationships between lumbar lordosis and pelvic parameters have been similarly shown to have a profound impact on HRQOL results. ${ }^{2}$ Traditionally, adult deformity surgery has usually involved an extensive open operation using a combination of spinal instrumentation, interbody fusion techniques, and osteotomies to achieve the desired spinal alignment. Substantial improvements in clinical symptoms have been achieved with these techniques, but the rate of perioperative complications and the prolonged recovery periods have been daunting. ${ }^{9}$ Even with these extensive procedures, failure to achieve ideal spinal alignment has been relatively common. ${ }^{5}$

Minimally invasive spinal fusion techniques have been achieving increasing popularity. Several studies have demonstrated shorter hospital stays, less pain, a lower perioperative incidence of certain complications, and faster recovery compared with traditional open techniques. ${ }^{6,10}$ There is little evidence that long-term results are substantially different between open and minimally invasive fusion procedures, with certain complications occurring more frequently in the minimally invasive fusion cohorts. ${ }^{6,10}$ Most surgeons recognize that minimally invasive fusion techniques are an acceptable option for the treatment of a spectrum of conditions requiring 1- or 2-level lumbar instrumentation and fusion. Because minimally invasive spinal procedures have demonstrated a reduction in certain perioperative complications, such as blood loss, infection, and paraspinal muscle damage, a number of investigators have begun attempting minimally invasive techniques for the correction of adult spinal deformity.

The initial presentations detailing the results of the use of minimally invasive spinal deformity correction techniques began in 2001. The initial presentations of these results detailed reduced blood loss, shorter hospital stays, and less perioperative pain compared with what one generally expected from open deformity correction procedures. Peer-reviewed publications of minimally invasive spinal deformity techniques were published starting in 2008, but most represented retrospective case series containing a limited number of patients and focused on operative duration, blood loss, perioperative complications, and hospital stay. Most of these early reports contained minimal HRQOL data and the follow-up duration was extremely short. Over the last 2 years, series have included greater numbers of patients and more HRQOL data but have offered limited radiographic evaluation. There has been a lack of consistent follow-up greater than 2 years and an absence of analysis of the sagittal vertical axis, pelvic tilt, and the relationship between lumbar lordosis and pelvic incidence. ${ }^{2}$ This lack of data has made direct comparison of minimally invasive and open deformity techniques difficult. A major concern following review of the cases included in the various reported minimally invasive case series is the general failure to restore normal sagittal alignment and adequate lumbar lordosis. Until now, there has been no prospective series with greater than 2 years of follow-up containing both HRQOL data and a contemporary radiographic evaluation of spinal deformity.

The article "Improvement of sagittal balance and lumbar lordosis following less invasive adult spinal deformity surgery with expandable cages and percutaneous instrumentation," written by Wang, ${ }^{11}$ is the first article to evaluate the radiographic outcomes of minimally invasive spinal deformity correction that approaches some of the standards used to assess open surgery. The study included 25 patients who have mild to moderate scoliotic curves with relatively mild sagittal imbalance (preoperative sagittal vertical axis $7.4 \pm 4.9 \mathrm{~cm}$ ). Unlike prior minimally invasive spinal deformity studies, 36-in standing radiographs were consistently used to assess spinal alignment, but pelvic parameters were not included. Global lumbar lordosis was determined by measuring the sagittal angulation between lines drawn parallel to the upper endplates of L-1 and S-1. The results of this study demonstrate that minimally invasive spinal techniques can adequately improve coronal deformity and sagittal balance as well as enhance lumbar lordosis. These radiographic results appear comparable with those of open surgery in these patients with these relatively mild deformities. 
There are several disappointing aspects of Dr. Wang's article. There is a shorter follow-up period than is common for clinical and radiographic deformity outcome evaluation; a minimum follow-up of 12 months was used instead of 24 months. Despite the CT-based analysis of fusion, numerous deformity series with long-term followup have demonstrated pseudarthrosis and loss of alignment as many as 5 years after the surgical procedure. The radiographic evaluation in the present study failed to include analysis of the pelvic parameters and the impact of the surgery on optimizing pelvic tilt and restoring lumbar lordosis (that is, pelvic incidence relationship). It has been demonstrated that although adequate sagittal alignment has been restored, the failure to correct pelvic tilt can result in poorer outcomes ${ }^{2}$ because compensatory pelvic retroversion (increased pelvic tilt) can readily mask inadequately corrected sagittal spinal alignment. Although this article focuses on changes in radiographic parameters, the failure to include any HRQOL data makes it impossible to correlate clinical and radiographic outcomes.

Although the author presents the approach in his series as "minimally invasive," careful review of the technique description would instead suggest that, in reality, it is more of a hybrid procedure that is far closer to traditional open approaches than truly being minimally invasive. The author's technique involves full takedown of the muscle and other soft-tissue structures on the side of the fractional curve, as would be the case for a traditional open approach. In addition, multilevel radical facetectomies and nerve root exposures are performed through this exposure to enable multilevel transforaminal lumbar interbody fusions. Indeed, Dr. Wang's approach is remarkably similar in exposure and tissue disruption to that of multilevel open transforaminal lumbar interbody fusions, albeit labeled as minimally invasive.

Furthermore, although the author indicates that the soft tissue are "preserved" on the contralateral side of the spine and that the contralateral side is "not exposed," in reality, review of the technique clearly demonstrates that there is substantial disruption of the muscle and fascia on this side as well. Multiple disruptions of the fascia and muscle are created for placement of the pedicle screws. In addition, the author states that fusion is achieved on this side of the spine through "decorticating the lamina and facet joint through the same fascial incision used for screw placement." To decorticate the lamina and lateral mass at each level of the spine, the author must be substantially disrupting the overlying muscle to gain access to the bony surfaces. Although the label "minimally invasive" is undoubtedly more appealing for patients and marketing, it should be questioned whether what the author has described is simply a modest variation of traditional open techniques that has been labeled as a minimally invasive approach.

Placement of pedicle screws without full exposure of the bony anatomy typically involves use of imaging, including fluoroscopy, intraoperative CT navigation, or a combination of these modalities. The author neglects to report the amount of radiation exposure incurred by the surgeon or the patient. Use of fluoroscopy typically requires the surgeon to be in close proximity to the radia- tion source, and the impact of the cumulative dose over multiple cases remains unclear. ${ }^{1,8}$ Intraoperative CT navigation has emerged as an alternative to fluoroscopy, and, although this may substantially reduce the exposure to the surgeon, it may substantially increase the exposure to the patient.

Although minimally invasive approaches are often touted for their reduced complication rates, careful review of the author's report suggests that his complication rate is comparable to that of traditional open approaches. In the acute hospitalization period alone, there was a $24 \%$ complication rate, including new neurological deficit, acute coronary syndrome, and multiple instrumentationrelated issues, 3 of which required return of the patients to the operating room to undergo revision.

Considering data on minimally invasive spinal deformity correction have been presented at national and international meetings for over 20 years, the failure to present a clinical series using the comprehensive battery of clinical and radiographic tools available to the spine clinician is concerning. All surgeons treating adult spinal deformity desire to optimize surgical strategies to provide the best clinical and radiographic outcomes, reduce complications, and achieve durable results. For minimally invasive spinal deformity surgery to achieve more widespread acceptance, a study needs to be performed that meets the standards used for evaluating clinical series of adult deformity surgery. While this study is a step in the right direction, it does not meet these standards.

(http://thejns.org/doi/abs/10.3171/2012.9.SPINE12335)

\section{Disclosure}

Dr. Shaffrey consults for Biomet, DePuy, Globus, and NuVasive; is employed by Medtronic; and receives royalties from Biomet and Medtronic. Dr. Smith consults for Biomet, DePuy, and Medtronic; he has received an honorarium from Globus for teaching a spine course.

\section{References}

1. Abdullah KG, Bishop FS, Lubelski D, Steinmetz MP, Benzel EC, Mroz TE: Radiation exposure to the spine surgeon in lumbar and thoracolumbar fusions with the use of an intraoperative computed tomographic 3-dimensional imaging system. Spine (Phila Pa 1976) 37:E1074-E1078, 2012

2. Ames CP, Smith JS, Scheer JK, Bess S, Bederman SS, Deviren $\mathrm{V}$, et al: Impact of spinopelvic alignment on decision making in deformity surgery in adults. A review. J Neurosurg Spine 16:547-564, 2012

3. Blondel B, Schwab F, Ungar B, Smith J, Bridwell K, Glassman $\mathrm{S}$, et al: Impact of magnitude and percentage of global sagittal plane correction on health-related quality of life at 2-years follow-up. Neurosurgery 71:341-348, 2012

4. Glassman SD, Dimar JR, Carreon LY, Campbell MJ, Puno RM, Johnson JR: Initial fusion rates with recombinant human bone morphogenetic protein-2/compression resistant matrix and a hydroxyapatite and tricalcium phosphate/collagen carrier in posterolateral spinal fusion. Spine (Phila Pa 1976) 30:1694-1698, 2005

5. Lafage V, Smith JS, Bess S, Schwab FJ, Ames CP, Klineberg E, et al: Sagittal spino-pelvic alignment failures following three column thoracic osteotomy for adult spinal deformity. Eur Spine J 21:698-704, 2012

6. Park Y,Ha JW: Comparison of one-level posterior lumbar inter- 


\section{Editorial}

body fusion performed with a minimally invasive approach or a traditional open approach. Spine (Phila Pa 1976) 32:537-543, 2007

7. Schwab F, Patel A, Ungar B, Farcy JP, Lafage V: Adult spinal deformity-postoperative standing imbalance: how much can you tolerate? An overview of key parameters in assessing alignment and planning corrective surgery. Spine (Phila Pa 1976) 35:2224-2231, 2010

8. Smith HE, Welsch MD, Sasso RC, Vaccaro AR: Comparison of radiation exposure in lumbar pedicle screw placement with fluoroscopy vs computer-assisted image guidance with intraoperative three-dimensional imaging. J Spinal Cord Med 31:532-537, 2008

9. Smith JS, Shaffrey CI, Glassman SD, Berven SH, Schwab FJ, Hamill CL, et al: Risk-benefit assessment of surgery for adult scoliosis: an analysis based on patient age. Spine (Phila Pa 1976) 36:817-824, 2011

10. Wang J, Zhou Y, Zhang ZF, Li CQ, Zheng WJ, Liu J: Comparison of one-level minimally invasive and open transforaminal lumbar interbody fusion in degenerative and isthmic spondylolisthesis grades 1 and 2. Eur Spine J 19:1780-1784, 2010

11. Wang MY: Improvement of sagittal balance and lumbar lordosis following less invasive adult spinal deformity surgery with expandable cages and percutaneous instrumentation. Clinical article. J Neurosurg Spine [epub ahead of print October 26, 2012. DOI: 10.3171/2012.9.SPINE111081]

\section{Response}

Michael Y. WANG, M.D.

Department of Neurological Surgery, University of Miami Miller School of Medicine, Miami, Florida

I thank Drs. Smith and Shaffrey for their insightful comments on my article "Improvement of sagittal balance and lumbar lordosis following less invasive adult spinal deformity surgery with expandable cages and percutaneous instrumentation." Their contributions to the field of spinal deformity have been significant, and I am in full agreement with all of their comments and criticisms.

The reality has been that the management of adult spinal deformity is a complex and evolving field, with many current concepts having only recently been validated. Nevertheless, the burgeoning population of elderly Americans mandates that we, as their physicians, find safer and more effective solutions for their problems, a matter that is far from trivial. Part of the problem with the development of techniques for minimally invasive spinal deformity surgery has been that lack of dialogue between surgeons who would classify themselves as specialists in deformity surgery and those who would classify themselves as specialists in minimally invasive spinal surgery.
One only needs to see the roster of meeting attendees of either subspecialty to see that there is minimal overlap. Indeed, there are few surgeons who would appropriately consider them to be true opinion leaders of both camps. This lack of dialogue has resulted in an unnecessary need to reinvent the wheel, sometimes in a suboptimal fashion. Many examples of how this has occurred are highlighted in my paper. For example, a lack of respect for the fractional curve or sagittal balance has no doubt led to countless cases in which a patient may have been poorly served by a minimally invasive operation.

The concept of what constitutes a minimally invasive approach itself is now being re-explored. Most minimally invasive surgery thought leaders would agree that the length of the skin incision may be the most unimportant determinant of "invasiveness." Previous erroneous concepts led to the idea that performing a surgery through a tubular retractor made a procedure less invasive. The end result were several Class I/IIa level studies showing that traditional microdiscectomy is equal or superior to minimally invasive surgical tubular discectomy. Thus, Drs. Smith and Shaffrey are absolutely correct in their categorization of the technique described in my paper as a hybrid. Adherence to the principal goals of deformity surgery mandates such an approach with current technology.

This technique was developed in response to the plethora of minimally invasive spinal deformity surgeries being performed across the US - namely, a lateral multilevel interbody fusion followed by placement of percutaneous screws. While that procedure represented a major advance, it fails in a number of respects: 1) it cannot address all levels of the spine; 2) it fails to adequately restore sagittal balance without additional extremely invasive additions; 3 ) it cannot address lumbosacral fractional curve problems; and 4) it is a surgery that is performed in 2 stages, prolonging anesthesia time in a compromised patient population. Furthermore, we have yet to see a publication of such surgeries being performed to treat severe deformities in either the coronal or sagittal plane.

Ultimately, all surgical techniques, including the one I describe, will need to evolve to better serve patients. The deformed, geriatric spine poses many challenges to the surgeon. A decade ago most surgeons would be reluctant to attempt any deformity surgery in an octogenarian. However, advances in surgical technique, anesthesia management, and postoperative care are now moving the needle in the right direction.

Please include this information when citing this paper: published online October 26, 2012; DOI: 10.3171/2012.9.SPINE12335. 